\title{
VEGETACIÓN ACUÁTICA Y SEMIACUÁTICA EN TRES RIOS ALTOANDINOS Y SU RELACIÓN CON LA FISICOQUÍMICA DEL AGUA
}

\author{
Cristian Botero-Álvarez ${ }^{12}$, Yimmy Montoya-Moreno², Néstor Jaime Aguirre-Ramírez ${ }^{23}$, Fabio de Jesús \\ Vélez-Macias ${ }^{23}$, Esnedy Hernández Atilano $^{23}$ \\ ${ }^{1}$ Estudiante Maestría en Ingeniería Ambiental. \\ 2 Profesor Universidad de Antioquia. \\ 3 Investigador grupo GeoLimna, Facultad de Ingeniería, Universidad de Antioquia. \\ Correo de correspondencia: cristianboteroa@gmail.com
}

\section{RESUMEN}

Los ríos altoandinos presentan presiones antrópicas por la necesidad de abastecimiento de agua debido al crecimiento demográfico. En ese sentido, los elementos bióticos asociados a estos sistemas como la vegetación acuática y semiacuática, pueden verse afectados dados los cambios que la actividad humana ocasiona sobre las corrientes naturales de montaña. La presente investigación se desarrolló en tres ríos, en jurisdicción del municipio de El Carmen de Viboral en el departamento de Antioquia, se establecieron cuatro muestreos para las tres estaciones, se midieron algunas variables fisicoquímicas para determinar su influencia sobre la estructura de la comunidad de hidrófitos. Se encontró que las variables más influyentes sobre la vegetación fueron caudal, $\mathrm{pH}$, conductividad eléctrica, nutrientes, temperatura del agua y turbiedad. Por lo anterior se corroboró la hipótesis planteada, de que las variables fisicoquímicas influyen en la distribución de plantas acuáticas en los ríos andinos.

Palabras clave: Plantas Acuáticas; Fisicoquímica; Ríos; Limnología.

Recibido: 30 de Marzo de 2020. Aceptado: 29 de Mayo de 2020.

Received: March 30, 2020. Accepted: May 29, 2020.

\section{AQUATIC AND SEMIACUATIC PLANTS IN THREE HIGH ANDEAN RIVERS AND ITS RELATIONSHIP WITH WATER PHYSICOCHEMISTRY}

\begin{abstract}
High Andean Rivers have anthropic pressures due to the need for water supply due to population growth. Thus, the biotic elements associated with these systems, such as aquatic and semiaquatic vegetation, may be affected by the changes that human activity causes on natural mountain currents. This research was carried out in three rivers, in El Carmen de Viboral municipality, Antioquia department, four samples were established for the three stations, some physicochemical variables were measured to determine their influence on the structure of the hydrophyte community. In this investigation it was found that the most influence on variables for vegetation were flow, $\mathrm{pH}$, electrical conductivity, nutrients, water temperature and turbidity. Due to the above, the hypothesis that the physicochemical variables influence the distribution of aquatic plants in the Andean rivers was corroborated.
\end{abstract}

Keywords: Aquatic plants; Physicochemical; Rivers; Limnology

Cómo citar este artículo: C. Botero, Y. Montoya, N. Aguirre, F. Vélez, E. Hernández. "Vegetación acuática y semiacuática en tres rios altoandinos y su relación con la fisicoquímica del agua", vol.16, no.31 pp.138-150, 2020.

DOI:

10.33571/rpolitec.v16n31a10 


\section{INTRODUCCIÓN}

Colombia en su división política cuenta con 33 departamentos y 1051 municipios; 10 de estos albergan el $70 \%$ de la población [1] y generan demanda sobre el recurso hídrico. Un caso a partir del cual se puede analizar el proceso y problemáticas locales asociadas con la demanda de agua, es representado por el Oriente Antioqueño, las proyecciones de población del Plan de Gestión Ambiental Regional CORNARE del año 2014 indican que para el año 2034, la región contará con 796.078 habitantes, lo que se traduce en un incremento aproximado del $15 \%$ en relación con el año 2020, donde se estima una población de 681.739 habitantes [2]. Este crecimiento demográfico y las actividades relacionadas con el desarrollo de las comunidades como las labores domésticas, industriales, agrícolas, entre otras [3], ha llevado a generar preocupación por la disminución en cantidad y calidad del recurso hídrico, lo cual genera el análisis de conflictos sociales por el acceso al recurso y la disponibilidad del agua por una distribución poco equitativa [4].

Así las cosas, se hace necesario ahondar en el estudio de los ríos andinos en la zona del Oriente Antioqueño, dichos sistemas acuáticos se ven afectados por este crecimiento demográfico. En términos físicos, estos ríos presentan condiciones particulares en el relieve, la topografía, tipos y usos de suelo; así, los ríos alto andinos muestran gran variabilidad en los caudales, aumentando en su magnitud a medida que avanza la red hídrica, además de sumar los cambios topográficos del terreno principalmente altitud y pendiente [5], adicionalmente la disminución de lámina de agua para aprovisionamiento del recurso hídrico en sus diversos usos.

Esta variabilidad física de las corrientes, incide sobre los componentes fisicoquímicos, lo que genera fluctuaciones en las concentraciones de estos; en ese sentido, variables como $\mathrm{pH}$, oxígeno disuelto, temperatura del agua, conductividad eléctrica y los nutrientes nitrógeno y fósforo, representan algunas de las variables más relevantes en los sistemas acuáticos y que a su vez, se ven alteradas en aumento o disminución en sus concentraciones debido a factores como contaminación por vertimientos de aguas residuales, pérdida de vegetación de ribera, entre otros factores [6], [7], [8], [9], [10].
Estas variaciones hidráulicas y fisicoquímicas mencionadas, alteran la composición, estructura, colonización y permanencia de las plantas acuáticas asociadas a las corrientes naturales, dado que estas comunidades biológicas, responden a cambios de dichas variables en el tiempo [11], [12].

Otra de las características que determina la calidad de los ríos altoandinos está relacionada con los usos de suelo, dado que los diversos usos pueden aportar contaminación ya sea por plaguicidas en actividades agrícolas o contaminación microbiológica por ganadería o aguas residuales domésticas, entre otras causas. Ahora bien, en Colombia los ríos altoandinos por sus diversos hábitats, variedad en las presiones ambientales y usos del suelo, forman parte de un escenario de interés con respecto a la diversidad en la comunidad florística y su respuesta a condiciones ambientales [13], [14]; este tipo de vegetación, es la que crece en zonas húmedas, principalmente ríos y lagos al interior de la masa de agua o parcialmente sumergida, otorgando a los sistemas acuáticos gran valor ecológico, dado que son biotopos fundamentales para albergar diversidad de fauna y microfauna como bacterias, hongos, macroinvertebrados acuáticos, e incluso perifiton y algas; adicionalmente estas plantas presentan características importantes en relación a la depuración de las aguas de forma natural y puede incluso llegar a regular en ciertos periodos climáticos los flujos de caudal; además de presentar una amplia distribución relacionada con sus tipos de crecimiento y condicionantes ambientales por lo que son organismos que presentan una alta diversidad biológica [15], [16], [17], [18], [19], [20].

Los ríos de alta montaña presentan características físicas y fisicoquímicas particulares, estas pueden condicionar la presencia y distribución comunitaria de los organismos que se allí de desarrollan, en este caso la vegetación acuática y semiacuática que cumple su ciclo de vida en estos ecosistemas húmedos y que puede presentar variación dados los cambios en la fisicoquímica de los ríos.

Es importante mencionar que las investigaciones relacionadas con este tipo de vegetación en Colombia son escasas, y en las que se encuentran disponibles, se hace un esfuerzo inicial en la identificación de los organismos, hábitos de vida y en pocos casos, se realizan algunas relaciones 
entre la distribución de los organismos y algunas variables ambientales [13], [14], [21], [22]. Sin embargo, los estudios no avanzan en aspectos importantes, como determinar la interacción de los cambios en la variabilidad fisicoquímica de los ríos y cómo esta puede afectar la distribución comunitaria de la vegetación acuática y riparia asociada.

Por lo anterior, se plantea la pregunta de investigación de cómo es la relación entre la estructura de la comunidad de plantas acuáticas y semiacuáticas con las variables fisicoquímicas en tres estaciones de ríos altoandinos; adicionalmente se plantea la hipótesis de que la estructura de la comunidad de hidrófitos está influenciada por las condiciones hidráulicas como caudal, además de la influencia en la presencia y distribución de las plantas por variables fisicoquímicas, entre las que se destacan la temperatura, la conductividad eléctrica y los nutrientes; para ello se propone como objetivo determinar la estructura de la comunidad de la vegetación acuática y semiacuática para tramos de las cabeceras de tres ríos andinos, y su posible relación con las algunas variables hidráulicas y fisicoquímicas de las fuentes hídricas.

\section{MATERIALES Y METODOS}

\section{Área de estudio:}

El área de estudio de las estaciones Cocorná, San Lorenzo y Guarinó asociadas a sus ríos del mismo nombre, se encuentran ubicadas en la zona noroccidental del municipio de El Carmen de Viboral, oriente del departamento de Antioquia en la parte alta de la cuenca del río Samaná Norte, afluente del río Magdalena.

En la figura 1 se identifican las tres estaciones de muestreo, estos tramos monitoreados se encuentran en zona de vida de bosque húmedo montano bajo (bh-MB) a unas altitudes de 2.289, 2.364 y 2.239 m.s.n.m. para las estaciones Cocorná, San Lorenzo y Guarinó respectivamente.

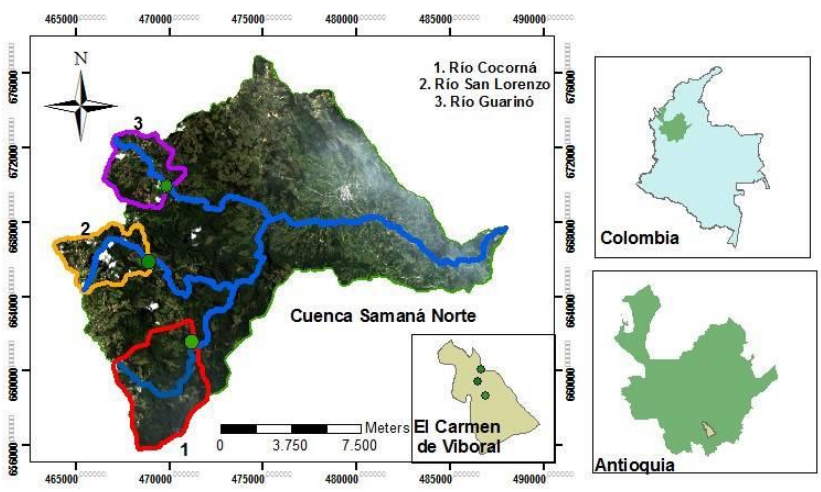

Fig. 1. Ubicación espacial de las estaciones de muestro (Colombia - Antioquia - El Carmen de Viboral - Cuenca Samaná Norte y estaciones).

Para cada una de las estaciones que se muestran en la figura 1 se evidencia una microcuenca asociada, para las cuales se tiene un área específica de drenaje. A continuación, en la tabla 1 se muestran las áreas de drenaje de cada una de las subcuencas de interés.

Tabla 1. Área de drenaje de subcuencas y porcentaje de usos de suelo

\begin{tabular}{ccccc}
\hline & \multirow{2}{*}{$\begin{array}{c}\text { Área de } \\
\text { drenaje }\end{array}$} & \multicolumn{3}{c}{ Porcentaje (\%) de uso de } \\
\cline { 3 - 5 } & suelo & \\
\cline { 3 - 5 } & $\left.\mathrm{km}^{2}\right)$ & Bosque & Pasto & $\begin{array}{c}\text { Suelo } \\
\text { Desnudo }\end{array}$ \\
\hline Cocorná & 22,1 & 73,3 & 13,0 & 13,7 \\
\hline $\begin{array}{c}\text { San } \\
\text { Lorenzo }\end{array}$ & 12,1 & 40,6 & 38,6 & 20,8 \\
\hline Guarinó & 12,3 & 28,9 & 50,7 & 20,4 \\
\hline
\end{tabular}

Se observa que el área del río Cocorná es la que más parche de bosque asociado tiene, por lo cual su intervención antrópica en las inmediaciones del cauce es la menor, además presenta un sustrato donde predominan rocas con diámetros entre 0,05 m y $1,5 \mathrm{~m}$; caso contrario para las estaciones San Lorenzo y Guarinó, que se evidenció en esta investigación un área de pastos y suelos desnudos mayor, por lo que su afectación en relación a la intervención humana es más alta, para la estación San Lorenzo el sustrato predominante es roca con diámetros entre los $0,05 \mathrm{~m}$ y $1,0 \mathrm{~m}$ y para la estación Guarinó se presenta un lecho donde dominan las arenas. 


\section{Metodología de campo, toma y medición de muestras fisicoquímicas y biológicas:}

El trabajo de campo se desarrolló en cuatro campañas de monitoreo en los días 1 y 2 de noviembre de 2018 (periodo lluvioso-Meso templado sin periodo seco), 12 y 13 de febrero de 2019 (periodo seco-Meso templado con periodo seco), 26 y 27 de marzo de 2019 (periodo secoMeso templado con periodo seco), 14 y 15 de mayo de 2019 (periodo lluvioso-Meso templado sin periodo seco). En cada estación se delimitó un tramo de $100 \mathrm{~m}$ longitudinalmente para la toma de muestras fisicoquímicas y biológicas.

Para la medición de variables fisicoquímicas se determinó el punto central del tramo donde el flujo presentara estabilidad; allí se midieron in situ con un equipo multiparamétrico WTW 3630 IDS temperatura del agua, conductividad eléctrica, sólidos totales disueltos, oxígeno disuelto, porcentaje de saturación de oxígeno y $\mathrm{pH}$; para la turbiedad se utilizó un HACH 2100Qis; también se determinó $\mathrm{CO}_{2}$ disuelto en el agua por medio de titulación con $\mathrm{NaOH}$ con una normalidad de $0,01 \mathrm{~N}$, es de resaltar que todos los equipos fueron calibrados antes de comenzar la medición.

Variables como fósforo total, alcalinidad total, color aparente, color verdadero, nitrógeno total Kjeldahl, sólidos suspendidos totales, sólidos totales, coliformes termotolerantes, coliformes totales y Escherichia coli fueron medidas en el Laboratorio de Estudios Ambientales y GDCON, ambos certificados por el IDEAM adscritos a la Universidad de Antioquia.

Las variables restantes, DQO, dureza cálcica y magnésica, ortofosfatos, nitritos, nitratos, nitrógeno amoniacal se midieron con kits Nanocolor y Visocolor, para la lectura de datos de se empleó un fotómetro PF-12 Macherey Nagel. En el caso de variables como nitritos, nitratos y ortofosfatos, se empleó un kit de reactivos $\mathrm{HACH}$ y para la medición de los datos, un espectrofotómetro $\mathrm{HACH}$ ODYSSEY DR2500, estos equipos pertenecen al grupo de investigación GeoLimna y el Laboratorio de Hidrobiología Sanitaria de la Universidad de Antioquia.

Para el levantamiento de las variables físicas como profundidad del cauce, ancho de sección y caudal, se emplearon cintas métricas, profundímetro, y correntómetro Global Water Flow Probe para medición de velocidades; para el lecho se hizo una caracterización visual y tener un referente cualitativo del mismo.

Para la toma de muestras biológicas, dentro del tramo de $100 \mathrm{~m}$ definido, este fue dividido en cinco secciones de $20 \mathrm{~m}$. En cada una de las líneas se tomaron muestras de los organismos en la margen derecha en la zona de transición agua - tierra, centro de la corriente y margen izquierda en la zona de transición agua - tierra, el muestreo se realizó en este orden en todos los monitoreos; para la medición de los organismos en los tres sitios, se siguió la metodología [23], se dispuso de un cuadrante de $1 \mathrm{~m}$ de lado ( $1 \mathrm{~m}^{2}$ en total); en cada una de estos cuadrantes se tomaron fotografias y registro vivo del número de individuos por taxa; en algunos casos fue necesario tomar muestras de los organismos de difícil identificación para analizar con la ayuda de claves taxonómicas [17], [18], [22], [24], [25] y la asesoría de un experto botánico del herbario de la Universidad de Antioquia.

Para la conservación de la vegetación, siguiendo las recomendaciones de [26], se utilizaron bolsas herméticas con una solución de alcohol al $70 \%$; la medición de la biomasa se desarrolló con una metodología propuesta, en esta se midió el peso húmedo de las plantas una vez con réplica para tener una masa promedio en $\mathrm{kg}$, para tomar las plantas se media la porción de área que esta ocupaba en el cuadrante de $1 \mathrm{~m}^{2}$ y se registraba en una tabla; posteriormente con las fotografias tomadas y el software ImageJ se media el área que ocupaban cada una de las plantas y con relación a esa área se daba el peso en los demás cuadrantes.

La determinación de la estructura de la comunidad de vegetación acuática y semiacuática se hizo en términos de algunos índices bióticos como Diversidad de Shannon Weaver, Dominancia de Simpson, Riqueza de especies, Equidad de Pielou y Abundancia asociada al número total de individuos, además de la biomasa en peso húmedo [27], [28], [29].

\section{Análisis estadístico:}

Se desarrolló un análisis descriptivo con medidas de tendencia central y de dispersión para conocer el comportamiento de las variables de interes y cuales de ellas presentaban mayor variabilidad, además de verificar si estas se ajustaban a una distribución normal; para comprobar la normalidad en los datos se aplicaron las pruebas de Shapiro Wilk (para muestras $<50$ observaciones) para las 
variables fisicoquímicas y de Kolmogorov-Smirnoff (para muestras > 50 observaciones) para las variables biológicas.

Posteriormente se corrió un Análisis de Componentes de Varianza - ACV; con este se determinó si los factores muestreo $(1,2,3,4)$ o estación (Cocorná, San Lorenzo y Guarinó) presentaban mayor influencia sobre la variabilidad y significancia de las variables. Teniendo este análisis, para los cuales se obtuvo una influencia > $30 \%$ del factor sobre la variable en el ACV, se elaboró una tabla con las variables y se desarrolló un Análisis de Varianza de una vía con una prueba no paramétrica de Kruskal-Wallis. En ese sentido, se redujo la dimensión del número de variables considerando tres criterios básicos; la sólidez de las observaciones, donde se evidenciaba que la variable o sus datos faltantes no superara el $75 \%$ del total de datos; la significancia en la variabilidad teniendo en cuenta el análisis descriptivo; y la influencia del medio acuático sobre las plantas desde una mirada ecosistémica.

Al final, se desarrolló un análisis multivariado para conocer qué variables tenían mayor influencia sobre la estructura de las plantas. Para el análisis, primero se corrió un Análisis de Correspondencia sin Tendencia - DCA para saber que tipo de prueba podría utilizarse, para un Análisis de Correspondencia Canónica - CCA la longitud del gradiente debía ser $>3,5$ y para un Análisis de Redundancia - RDA esta misma debía ser < 2,5, con esta prueba se corroboró que el RDA era el más adecuado, dado que el gradiente ambiental fue $<2,5[30]$

Todos los análisis estadísticos fueron trabajando con los software R3.6.1 para los cuales fueron utilizados los paquetes lattice, grid, cwhmisc, permute y vegan; además del software Statgraphics Centurion XVI.II.

\section{RESULTADOS}

\section{Caracterización fisicoquímica}

Dado el número de muestreos desarrollados, se obtuvo un total de 12 observaciones para la consolidación de la matriz fisicoquímica. En la tabla 2 , se muestran todas las variables fisicoquímicas que fueron medidas en el trabajo de campo. De estas variables se procedió a descartar las que tuvieran menor relevancia en términos de variabilidad dentro del análisis.

En la tabla 3 se muestran las variables fisicoquímicas que fueron establecidas como matriz de variables explicativas dentro del análisis posterior.

Tabla 3. Medidas de tendencia central y dispersión de variables fisicoquímicas

\begin{tabular}{ccccc}
\hline Variable & Promedio & Min & Max & $\begin{array}{c}\text { C.V. } \\
(\%)\end{array}$ \\
\hline $\begin{array}{c}\text { Caudal medio } \\
\left(\mathrm{m}^{3} / \mathrm{s}\right)\end{array}$ & 1,18 & 0,23 & 4,34 & 105,23 \\
\hline $\begin{array}{c}\mathrm{pH}(\text { Unidades } \\
\text { de } \mathrm{pH})\end{array}$ & 6,62 & 5,09 & 7,31 & 10,59 \\
\hline $\begin{array}{c}\text { Temperatura } \\
\text { del Agua }\left({ }^{\circ} \mathrm{C}\right)\end{array}$ & 16,42 & 14,70 & 18,70 & 8,22 \\
\hline $\begin{array}{c}\text { Conductividad } \\
\text { eléctrica } \\
(\mu \mathrm{S} / \mathrm{cm})\end{array}$ & 21,09 & 6,27 & 41,10 & 53,84 \\
\hline $\begin{array}{c}\text { Turbiedad } \\
(\text { UNT) }\end{array}$ & 6,75 & 1,33 & 28,30 & 119,72 \\
\hline $\begin{array}{c}\text { Nitrógeno } \\
\text { total soluble } \\
\text { (mg/L N) }\end{array}$ & 0,83 & 0,00 & 2,68 & 104,79 \\
\hline $\begin{array}{c}\text { Ortofosfatos } \\
\text { (mg/l PO } 4^{3-} \text {-P) }\end{array}$ & 0,26 & 0,00 & 2,19 & 240,02 \\
\hline
\end{tabular}

La tabla 3 muestra las variables que cumplieron los tres criterios de selección que se propusieron en la metodología, en esta tabla se evidencia que variables como caudal, conductividad eléctrica, turbiedad, nitrógeno total soluble y ortofosfatos presentaron un \% de variación $>40 \%$ por lo cual dentro de los muestreos fueron observaciones con mucha variabilidad, mientras que variables como $\mathrm{pH}$, y temperatura del agua permanecieron muy estables. Dicha variabilidad y distancias entre máximos y mínimos para cada variable estuvo relacionada con los periodos climáticos en que se desarrollaron los muestreos y las diferencias entre las características de las tres estaciones en cuestión. 
Tabla 2. Matriz de variables fisicoquímicas completa

\begin{tabular}{|c|c|c|c|c|c|c|c|c|c|c|c|c|}
\hline Muestreo & Río & $\begin{array}{c}\text { pH } \\
\text { (Unidades } \\
\text { de pH) }\end{array}$ & $\begin{array}{c}\text { Oxígeno } \\
\text { Disuelto } \\
\text { (ppm) }\end{array}$ & $\begin{array}{c}\% \\
\text { saturación } \\
(\%)\end{array}$ & $\begin{array}{l}\text { Temperatura } \\
\text { del Agua } \\
\left({ }^{\circ} \mathrm{C}\right)\end{array}$ & $\begin{array}{l}\text { Conductividad } \\
\text { eléctrica } \\
(\mu \mathrm{S} / \mathrm{cm})\end{array}$ & $\begin{array}{c}\text { Sólidos } \\
\text { Totales } \\
\text { Disueltos } \\
\text { (mg/L) }\end{array}$ & $\begin{array}{c}\mathrm{CO}_{2} \\
(\mathrm{mg} / \mathrm{L})\end{array}$ & $\begin{array}{c}\text { Turbiedad } \\
\text { (UNT) }\end{array}$ & $\begin{array}{c}\mathrm{DQO} \\
(\mathrm{mg} / \mathrm{L} \\
\left.\mathrm{O}_{2}\right)\end{array}$ & $\begin{array}{c}\text { Dureza } \\
\text { (mg/L } \\
\mathrm{Ca})\end{array}$ & $\begin{array}{c}\text { Dureza } \\
\text { (mg/L } \\
\text { Mg) }\end{array}$ \\
\hline \multirow{3}{*}{1} & Cocorná & 7,14 & 7,95 & 106,0 & 16,15 & 13,10 & 13,0 & 0,00 & 1,33 & $<\mathrm{LCM}$ & $<\mathrm{LCM}$ & 14,00 \\
\hline & San Lorenzo & 6,70 & 7,67 & 103,5 & 16,10 & 20,00 & 20,0 & 0,00 & 1,97 & $<\mathrm{LCM}$ & $<\mathrm{LCM}$ & 6,00 \\
\hline & Guarinó & 7,31 & 7,64 & 105,3 & 17,80 & 27,80 & 28,0 & 0,00 & 4,39 & $<$ LCM & $<\mathrm{LCM}$ & 26,00 \\
\hline \multirow{3}{*}{2} & Cocorná & 5,30 & 8,36 & 108,7 & 14,85 & 8,05 & 8,0 & 5,28 & 2,62 & $<$ LCM & $<$ LCM & 6,00 \\
\hline & San Lorenzo & 6,79 & 7,58 & 102,7 & 16,50 & 20,80 & 21,0 & 5,94 & 5,30 & $<$ LCM & $<\mathrm{LCM}$ & 24,00 \\
\hline & Guarinó & 7,06 & 7,84 & 109,5 & 18,70 & 41,10 & 41,0 & 3,52 & 9,96 & $<L C M$ & $<\mathrm{LCM}$ & 48,00 \\
\hline \multirow{3}{*}{3} & Cocorná & 7,05 & 8,09 & 104,2 & 14,70 & 9,30 & 9,5 & 1,32 & 1,43 & $<\mathrm{LCM}$ & $<\mathrm{LCM}$ & $<\mathrm{LCM}$ \\
\hline & San Lorenzo & 6,93 & 7,53 & 101,2 & 15,90 & 21,00 & 21,0 & 0,66 & 2,56 & 13,00 & $<\mathrm{LCM}$ & $<\mathrm{LCM}$ \\
\hline & Guarinó & 6,77 & 7,35 & 100,6 & 17,60 & 39,90 & 40,0 & 1,32 & 16,50 & 4,00 & $<$ LCM & 40,00 \\
\hline \multirow{3}{*}{4} & Cocorná & 5,09 & 8,13 & 105,2 & 14,9 & 6,27 & 6,3 & 3,52 & 2,66 & 18,00 & $<\mathrm{LCM}$ & $<\mathrm{LCM}$ \\
\hline & San Lorenzo & 6,81 & 7,63 & 101,7 & 15,7 & 19,80 & 20,0 & 1,76 & 3,92 & $<$ LCM & $<\mathrm{LCM}$ & 21,00 \\
\hline & Guarinó & 6,52 & 7,26 & 99,9 & 18,1 & 26,00 & 26,0 & 3,52 & 28,30 & $<\mathrm{LCM}$ & $<\mathrm{LCM}$ & $<\mathrm{LCM}$ \\
\hline
\end{tabular}


Continuación Tabla 2. Matriz de variables fisicoquímicas completa

\begin{tabular}{|c|c|c|c|c|c|c|c|c|c|c|c|c|c|}
\hline $\begin{array}{l}\text { Muestr } \\
\text { eo }\end{array}$ & Río & $\begin{array}{l}\text { Color } \\
\text { aparen } \\
\text { te } \\
\text { (UPC) }\end{array}$ & $\begin{array}{l}\text { Color } \\
\text { verdad } \\
\text { ero } \\
\text { (UPC) }\end{array}$ & $\begin{array}{l}\text { Fósfo } \\
\text { ro } \\
\text { total } \\
(\mathrm{mg} / \mathrm{L} \\
\mathrm{P})\end{array}$ & $\begin{array}{c}\text { Alcalinid } \\
\text { ad total } \\
(\mathrm{mg} / \mathrm{L} \\
\left.\mathrm{CaCO}_{3}\right)\end{array}$ & $\begin{array}{l}\text { Sólidos } \\
\text { Suspendid } \\
\text { os Totales } \\
\text { (mg/L } \\
\text { SST) }\end{array}$ & $\begin{array}{l}\text { Sólid } \\
\text { os } \\
\text { Total } \\
\text { es } \\
\text { (mg/L } \\
\text { ST) }\end{array}$ & $\begin{array}{c}\text { Nitróge } \\
\text { no total } \\
\text { soluble } \\
\text { (mg/L } \\
\mathrm{N})\end{array}$ & $\begin{array}{c}\text { Nitróge } \\
\text { no } \\
\text { Kjenda } \\
\text { hl } \\
(\mathrm{mg} / \mathrm{L} \\
\mathrm{N})\end{array}$ & $\begin{array}{l}\text { Ortofosfat } \\
\text { os (mg/l } \\
\left.\mathrm{PO}_{4}{ }^{3-}-\mathrm{P}\right)\end{array}$ & $\begin{array}{c}\text { Coliformes } \\
\text { termotoleran } \\
\text { tes } \\
(\mathrm{NMP} / 100 \mathrm{~mL} \\
\text { ) }\end{array}$ & $\begin{array}{c}\text { Coliformes } \\
\text { totales } \\
\text { (NMP/100 } \\
\text { mL) }\end{array}$ & $\begin{array}{c}\text { E. coli } \\
\text { (NMP/100 } \\
\mathrm{mL})\end{array}$ \\
\hline \multirow{3}{*}{1} & Cocorná & 10 & N.D & 0,300 & 5,16 & 0 & 25 & 0,3640 & 0,666 & 0,0250 & 11 & 3260 & 9 \\
\hline & $\begin{array}{c}\text { San } \\
\text { Lorenzo }\end{array}$ & 5 & N.D & 0,500 & 6,69 & 2 & 28 & 0,4770 & 0,638 & 0,0040 & 30 & 1570 & 20 \\
\hline & Guarinó & 15 & N.D & N.D & 14,70 & 2 & 45 & 0,2020 & 0,851 & 0,0350 & 10 & 176 & 9 \\
\hline \multirow{3}{*}{2} & Cocorná & 67 & N.D & 0,020 & N.D & 3 & 32 & 0,0048 & 1,260 & 0,0033 & 34 & 980 & 53 \\
\hline & $\begin{array}{c}\text { San } \\
\text { Lorenzo }\end{array}$ & 28 & N.D & 0,030 & N.D & 4 & 45 & 0,0054 & 1,290 & 0,2087 & 131 & 2420 & 179 \\
\hline & Guarinó & 50 & N.D & 0,026 & N.D & 4 & 64 & 1,0896 & 1,370 & 0,0261 & 1733 & 5450 & 1090 \\
\hline \multirow{3}{*}{3} & Cocorná & 25 & 21,20 & 0,008 & 3,02 & 0 & 30 & 0,5899 & 1,170 & 0,0033 & 17 & 1733 & 96 \\
\hline & $\begin{array}{c}\text { San } \\
\text { Lorenzo }\end{array}$ & 15 & 7,83 & 0,008 & 7,30 & 2 & 32 & 0,7813 & 0,675 & 2,1914 & 1300 & 5480 & 650 \\
\hline & Guarinó & 25 & 9,41 & 0,024 & 17,00 & 14 & 71 & 0,2370 & 0,653 & 0,0457 & 172 & 2010 & 30 \\
\hline \multirow{3}{*}{4} & Cocorná & 55 & 54,40 & 0,008 & 0,50 & 0 & 26 & 2,6809 & 1,220 & 0,5707 & 20 & 2030 & 11 \\
\hline & $\begin{array}{c}\text { San } \\
\text { Lorenzo }\end{array}$ & 15 & 12,60 & 0,010 & 5,86 & 0 & 24 & 2,3026 & 0,319 & 0,0293 & 100 & 5200 & 50 \\
\hline & Guarinó & 40 & 23,20 & 0,056 & 10,00 & 13 & 44 & 1,2321 & 0,269 & 0,0026 & 100 & 5200 & 50 \\
\hline
\end{tabular}


La tabla 2 muestra información relevante para posteriores investigaciones, dado que como línea base aporta elementos para el entendimiento de la fisicoquímica de las estaciones que fueron objeto de esta investigación; dentro de las variables que aparecen con datos marcados como <LCM- Límite de cuantificación del método, implica que estas variables presentaros datos inferiores a los que podía reportar el equipo de medición; adicionalmente, las casillas N.D- No Dato, implica que allí no fue posible tener el valor de medición por errores en la toma de la muestra de agua en campo.

\section{Caracterización biológica}

Se determinaron un total de 22 taxa que estaban distribuidas por los tres sistemas acuáticos, estos organismos se presentaron de la siguiente manera.

Para la estación Cocorná se encontraron organismos de la familia Lycopodiaceae: Lycopodium sp.; Myrtaceae; Asteraceae: Ageratina sp.; Rubiaceaea: Gallium sp.; Cyperaceae: Cyperus sp., Eleocharis filiculmis; Oxalidaceae: Oxalis sp.; Dryopteridaceae: Elaphoglossum sp.; Thelypteridaceae: Thelypteris sp.; Blechnaceae: Blechnum cordatum y Marchantiaceae: Marchantia sp.

Para la estación San Lorenzo se encontraron organismos de la familia Cyperaceae: Cyperus mindorensis, Cyperus carex, Rhynchospora sp., Eleocharis filiculmis; Apiaceae: Hydrocotyle umbellata; Thelypteridaceae: Thelypteris sp.; Blechnaceae: Blechnum cordatum; Marchantiaceae: Marchantia sp. y Brachytheciaceae: Platyhypnidium aquaticum.

Para la estación Guarinó se encontraron organismos de la familia Cyperaceae: Eleocharis filiculmis; Apiaceae: Hydrocotyle umbellata, Centella asiatica; Juncaceae: Juncus microcephalus; Polygonaceae: Polygonum puntactum; Potamogetonaceae: Potamogeton paramoanus; Zingiberaceae : Hedychium coronarium; Thelypteridaceae: Thelypteris sp.; Marchantiaceae: Marchantia sp. y Brachytheciaceae: Platyhypnidium aquaticum.

Igual que para las variables fisicoquímicas, las variables biológicas respuesta se filtraron al tener en cuenta los mismos criterios, en la tabla 3 se muestran las cuatro variables respuesta que fueron utilizadas.
Tabla 4. Medidas de tendencia central y dispersión de variables biológicas

\begin{tabular}{ccccc}
\hline Variable & Promedio & Min & Max & $\begin{array}{c}\text { C.V. } \\
\%\end{array}$ \\
\hline $\begin{array}{c}\text { Diversidad } \\
\text { Shannon } \\
\text { Weaver }\end{array}$ & 1,26 & 0,77 & 2,02 & 42,40 \\
\hline $\begin{array}{c}\text { Riqueza de } \\
\text { especies }\end{array}$ & 8,00 & 5,00 & 11,00 & 22,00 \\
\hline $\begin{array}{c}\text { Abundancia } \\
\left(\text { Ind./m } / \mathrm{m}^{2}\right)\end{array}$ & 18,00 & 0,00 & 130,00 & 159,85 \\
\hline $\begin{array}{c}\text { Biomasa } \\
\text { total }\left(\mathrm{kg} / \mathrm{m}^{2}\right)\end{array}$ & 498,88 & 0,00 & 2972,41 & 131,88 \\
\hline
\end{tabular}

De la tabla anterior se evidencia un \% de variación bajo para la riqueza, variable que describe el número de taxa, este pudo haber sido bajo dado que para cada río en general se repitieron las mismas especies, pero haciendo una comparación entre las tres estaciones hubo algunas diferencias entre las mismas; mientras que las demás variables alcanzaron variaciones $>40 \%$ lo que implica que estas fluctuaron más entre muestreos y estaciones.

Con respecto a la misma tabla es importante mencionar que la presencia de organismos fue marcada por la siguiente tendencia; en el río Cocorná los organismos que mayor abundancia fueron las Myrtaceas, Eleocharis filiculmis, Oxalis $\mathrm{sp}$ y Thelypteris $\mathrm{sp}$; mientras que en el río San Lorenzo las Cyperaceas marcaron la riqueza pero en muy pocas abundancias; por último el río Guarinó, aunque presentó riqueza similar al San Lorenzo este tuvo la menor abundancia, incluso con muchos cuadrantes sin organismos, allí se evidenció abundancia de Hydrocotyle umbellata y Centella asiatica. Ahora comparando entre ríos, el río Cocorná presentó las mayores abundancias, segudo del San Lorenzo y el Guarinó respectivamente. En términos de Diversidad, el río San Lorenzo era el mas diverso, seguido de las otras dos corrientes.

\section{Análisis estadístico multivariado}

El Análisis de Redundancia-RDA arrojó un porcentaje de explicación del modelo $87,8 \%$, distribuidos en $47,8 \%$ para el componente 1 y $40,0 \%$ para el componente 2 . Adicional a esto el $\mathrm{R}^{2}$ es 1 por lo cual el modelo indica que toda la 
variabilidad de las variables biológicas está determinada por las variables fisicoquímicas del sistema, este ajuste es posible que se haya dado, teniendo en cuenta que el número de observaciones era bajo, por lo que toda esta variabilidad biológica se volcó sobre las observaciones fisicoquímicas existentes.

A continuación, en la figura 2 se muestra de manera gráfica los resultados del RDA.

Fig. 2. RDA - Entre variables fisicoquímicas y biológicas.

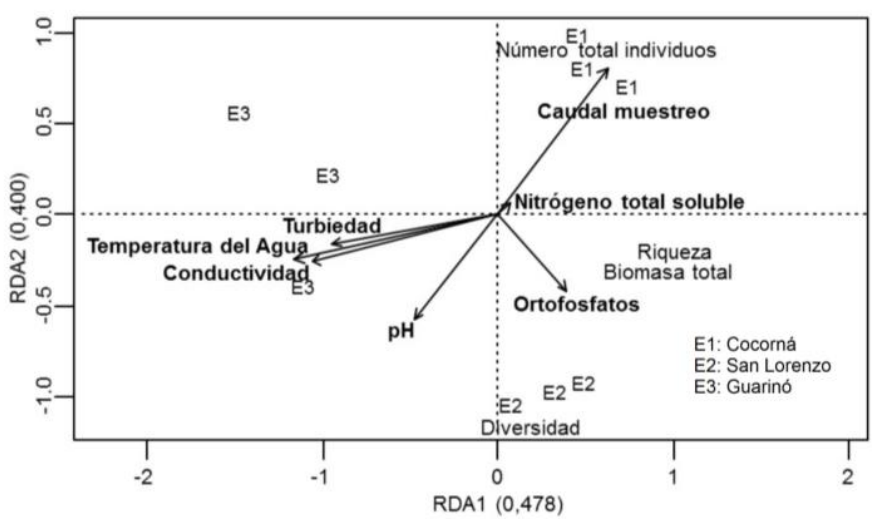

El análisis anterior muestra el peso o importancia que tiene cada variable fisicoquímica - explicativas, sobre cada variable biológica - respuestas, por lo anterior, se puede plantear que las relaciones encontradas pueden determinar la influencia de las variables fisicoquímicas sobre las plantas acuáticas. Al tomar en consideración el componente $1(47,8 \%)$ las variables que tuvieron una mayor influencia sobre biomasa y riqueza de las plantas acuáticas fueron temperatura, conductividad y turbiedad, de manera inversa, y nitrógeno total soluble de manera directa; y para el componente $2(40,0 \%)$ el caudal influyó de manera directa sobre la abundancia de los individuos y de manera inversa sobre la diversidad; mientras que el $\mathrm{pH}$ y los ortofosfatos influyeron de manera directa sobre la diversidad y de manera inversa sobre la abundancia.

\section{ANÁLISIS DE RESULTADOS Y DISCUSIÓN}

Según los resultados obtenidos se evidenció una separación importante entre las estaciones en relación con su hidráulica y estructura física, esto se debió principalmente a que el río Cocorná presenta un caudal mayor en relación con los otros dos sistemas de interés, además de sus diferencias en términos de lecho, dado que el río Cocorná presentó un sustrato con rocas de diámetro mucho mayor que los demás sistemas, mientras que el río Guarinó presentó un lecho con predominio de arenas.

Así mismo, se observaron variaciones en relación con las variables fisicoquímicas, aunque estas fueron más representativas entre sistemas que entre muestreos, caso particular el del río Guarinó, donde se presentaron los valores más altos de variables relacionadas con el transporte de sedimentos, como turbiedad, conductividad eléctrica, color aparente, sólidos suspendidos totales, entre otras. Lo que es favorecido por el predominio del sustrato arenoso en el cauce del río, sustrato que no es predominante en ríos de alta montaña, pero por los diversos usos del suelo aguas arriba y en inmediaciones de la estación, se ven alteradas las condiciones naturales de dicho sistema

Las variables fisicoquímicas medidas, presentan una relación importante con la conformación de la estructura biológica de interés, en otras investigaciones se ha evidenciado que variables como conductividad y temperatura, tipo de sustrato, nutrientes como nitrógeno y fósforo en sus formas iónicas disponibles, caudal y velocidad de la corriente, explican en gran medida la distribución y presencia de la vegetación acuática [14], [35], [37], dichas variables en el ecosistema natural influyen en el crecimiento de las plantas como el caso de los nutrientes, por otra parte variables como el caudal y temperatura condicionan la presencia o ausencia de algunos taxa.

En lo que respecta a la estructura biológica se evidenciaron cambios interesantes en la distribución de taxa asociados principalmente a cada estación. Dado que el río Cocorná presentó los valores más altos de abundancia, pero en relación con la diversidad el río San Lorenzo tuvo lo mayores valores, y por último el río Guarinó fue el sistema con valores mínimos de diversidad y número de individuos.

El tipo de sustrato condicionó la presencia de algunos individuos en la zona de ritral de cada sistema, caso particular del río Cocorná, al tener un sustrato rocoso, se observó de manera reiterada la colonización de especies como Ageratina sp y 
Eleocharis filiculmis; así mismo, para el río Guarinó que presentó un lecho principalmente arenoso, se evidenció la colonización por parte la especie acuática estricta Potamogeton paramoanus, como se ha encontrado en otras investigaciones [31].

Los cambios en las dinámicas del flujo asociados a variables como caudal pudieron afectar la distribución de las plantas acuáticas, dado que algunos organismos son sensibles a esos cambios, teniendo en cuenta que la morfología de dichos individuos no les permite adaptarse de manera adecuada a estos ambientes [32], [33], [34]. Se observaron diferencias de los tipos de organismos y su distribución espacial en la masa de agua, relacionadas con la abundancia y diversidad de los mismos en los ríos Cocorná, San Lorenzo y Guarinó; además de ello, investigaciones como [35] encontraron que los cambios de caudal pueden afectar la colonización de especies, por tanto, donde hay menores velocidades existe una mayor diversidad, lo que se comprobó con la presente investigación, dado que el río Cocorná presentó mayores velocidades y una menor diversidad de especies comparado con las demás estaciones.

De las variables fisicoquímicas se encontró que el nitrógeno, fósforo, temperatura del agua, $\mathrm{pH}$, conductividad eléctrica y alcalinidad, son algunas de las variables que mayor influencia tienen con el crecimiento de hidrófitos, lo cual está en concordancia con lo planteado por otras investigaciones [36], [37].

El Análisis de Redundancia-RDA mostró agrupaciones entre estaciones, por ejemplo, el río Cocorná tuvo afinidad con caudales altos, allí se presentó menor diversidad, pero mayor abundancia, esto debido a que el caudal funciona como presión ambiental sobre los individuos lo que dificulta su colonización y permanencia [35], lo contrario en el río San Lorenzo, donde se observó mayor diversidad, pero los individuos existentes no presentaron abundancias significativamente altas en comparación con el Cocorná. Por último, se observó que el río Guarinó tenía mayores magnitudes en las variables fisicoquímicas como conductividad, temperatura del agua y turbiedad, lo que pudo condicionar la poca presencia de vegetación acuática.

Se evidenció que variables como el caudal, afectaron la conformación de los organismos al influir en la presencia o ausencia de unas $u$ otras especies. Caso particular, de las especies Hydrocotyle umbellata y Centella asiatica que crecen en sistemas acuáticos leníticos o ríos con velocidades bajas [38], dichas especies fueron encontradas en el río Guarinó principalmente. Adicionalmente, la planta del género Oxalis sp que vive principalmente en sustrato rocoso y en bordes de ríos fue encontrada principalmente en el río Cocorná [39].

Es importante considerar que las plantas acuáticas pueden ser sensibles a los cambios en las condiciones ambientales [40], en ese sentido las variables fisicoquímicas como temperatura del agua, conductividad eléctrica, $\mathrm{pH}$, nitrógeno total soluble y ortofosfatos se relacionaron con la biomasa y riqueza de los organismos. Caso particular de algunos organismos, como los briofitos, Marchantia sp y Platyhypnidium aquaticum que necesitan grandes cantidades de agua para sobrevivir, además de la presencia de nutrientes como nitrógeno [36], [41], [42].

Otros individuos como los pertenecientes a la familia Cyperaceae encontrados en los muestreos, con mayor riqueza en el río San Lorenzo necesitan mayor cantidad de nutrientes para cumplir su ciclo de vida [43]. En contraste a lo reportado en [14] quienes expresan que a mayores valores de temperatura y conductividad se presentaron las mayores riquezas y abundancias, en la presente investigación se evidenció lo contrario, donde a mayores valores de temperatura y conductividad caso de la estación Guarinó, se encontró menor riqueza biológica, esto posiblemente por la diferencia en los usos de suelo en comparación con las demás estaciones de investigación.

\section{CONCLUSIONES}

La presencia, distribución espacial y tipo de vegetación acuática y semiacuática está influenciada de forma directa por variables físicas como tipo de sustrato, dado que las plantas pueden crecer más fácilmente en bases sólidas con algunas fisuras como las rocas del río Cocorná, y el caudal, teniendo en cuenta que su magnitud permite que algunas plantas pueden desarrollarse mejor, es el caso de las plantas de la familia Myrtaceae y una Cyperaceae (Eleocharis filiculmis) encontradas, que tienen unas raíces más sólidas, 
estas fueron muy abundantes en el río Cocorná, donde se presentaron los mayores caudales.

Las variables fisicoquímicas más importantes dentro del análisis fueron el $\mathrm{pH}$, conductividad, temperatura del agua, turbiedad y nutrientes; la variabilidad de estas se relacionó más con el tipo de río que con la campaña de muestreo. Por consiguiente, los cambios en la estructura de la comunidad de vegetación se asociaron en igual medida a los tipos de ríos. Así las cosas, y como se mencionó en la discusión, la fluctuación de estas variables fisicoquímicas permite que organismos como las plantas acuáticas encontradas puedan prosperar mejor en unos u otros ambientes.

La intervención antrópica, derivada principalmente en obras de captación para suministro de agua pueden disminuir de forma drástica la lámina de agua principalmente en las estaciones San Lorenzo y Guarinó, por lo que organismos como Cyperus $\mathrm{sp}$, Cyperus mindorensis, Cyperus carex, Rhynchospora sp, Oxalis sp, Hydrocotyle umbellata, Hydrocotyle asiatica, Juncus microcephalus y Polygonum puntactum que se encontraron en la banca principalmente podrían resultar afectadas por dicha pérdida de flujo, dado que los caudales naturales mantienen un nivel de agua que hace posible que los organismos puedan cumplir su ciclo de vida.

\section{AGRADECIMIENTOS}

Los autores agradecen a la Universidad de Antioquia y al municipio de El Carmen de Viboral por la financiación a esta investigación; al grupo de investigación GeoLimna; al laboratorio de Hidrobiología Sanitaria y Herbario de la Universidad de Antioquia; a la Dra. Diana Agudelo por sus aportes en el análisis estadístico; y por último a los investigadores amigos que apoyaron el proceso.

\section{REFERENCIAS BIBLIOGRÁFICAS}

[1] IANAS. La calidad de agua en Colombia, Red regional de Academias de Ciencias, 202-238, 2019

[2] CORNARE. PLAN DE GESTIÓN AMBIENTAL REGIONAL 2014-2032, El Santuario, 2014.

[3] Gastañaga, M. Agua, saneamiento y salud, Peru. Rev. Peru Med. Exp. Salud, 35(2):181-2, 2018
[4] Agudelo, R. El agua, Recuros estratégico del sigle XXI, Medellin, Revista Facultad Nacional de Salud Pública. 23(1):91-102, 2005.

[5] Segnini, S. Chacón, M., Caracterización fisicoquímica del hábitat interno y ribereño de rios andinos en la cordillera de Mérida, Venezuela, ECOTROPICOS 18(1):38-61, 2005.

[6] Pasquini, A.I., Lecomte, K.L., Depetris, P.J. Geoquímica de ríos de montaña en las Sierras Pampeanas: II. El río Los Reartes, sierra de Comenchingones, provincia de Córdoba, Revista de la Asociación Geológica Argentina, 59 (1): 129140, (2004).

[7] García-Alzate, C.A., Román-Valencia, C., Vanegas-Ríos, J.A., Arcila-Mesa, D.K. Análisis Fisicoquímico y Biológico Comparado en Dos Quebradas de Alta Montaña Neotropical, Revista de Investigaciones, Universidad del Quindio, 17(1): 57-80. ISSN 1794-631X, 2007.

[8] Villamarín, C., Prat, N., Rieradevall, M. Caracterización física, química e hidromorfológica de los ríos altoandinos tropicales de Ecuador y Perú, Lat. Am. J. Aquat. Res, 42(5): 1072-108, 2014.

[9] Benjumea-Hoyos, C.A., Suárez-Segura, M.A., Villabona-González, S.L. Variación espacial y temporal de nutrientes y total de sólidos en suspensión en la cuenca de un río de alta montaña tropical, Rev. Acad. Colomb. Cienc. Ex. Fis. Nat., 42(165):353-363, 2018.

[10] Galeano-Rendón, E., y Mancera-Rodríguez, N.J. Efectos de la deforestación sobre la diversidad y la estructura del ensamblaje de macroinvertebrados en cuatro quebradas Andinas en Colombia, Revista de Biología Tropical, 66(4), 2018.

[11] Martinez, L.F., Donato, J. Efectos del caudal sobre la colonización de algas en un río de alta montaña tropical (Boyacá, Colombia), Caldasia, 25(2): 337-354, 2003.

[12] Díaz-Quiroz, C., Rivera-Rendón, C.A. Diatomeas de pequeños ríos andinos y su utilización como indicadoras de condiciones ambientales, Caldasia, 26(2): 381-394, 2004.

[13] Schmidt-Mumm, U. Vegetación Acuática y Palustre de la Sabana de Bogotá y Plano del Río 
Ubaté, Bogotá, Colombia: Universidad Nacional de Colombia, 1998.

[14] Gómez-Rodríguez, A.M., ValderramaValderrama, L.T., Rivera-Rondón, C.A. Comunidades de macrófitas en ríos andinos: composición y relación con factores ambientales, Acta biol. Colomb, 22(1):45-58, 2017.

[15] U.S. EPA. Methods for Evaluating Wetland Condition: Using Vegetation to Assess Environmental Conditions in Wetlands. Office of Water, U.S. Environmental Protection Agency, Washington, DC., 2002.

[16] García Murillo, P. Estudio Palinológico del Género Potamogeton L. en la Península Ibérica, Bot. Complutensis. 18:79-91, 1993.

[17] Giménez, P.T. Guia visual de campo: Macrófitos vasculares de la cuenca del Ebro, España, 2009.

[18] Cirujano Bracamonte, S., Meco Molina, A., García Murillo, P., Chirino Argenta, M. Flora Acuática Española: Hidrófitos Vasculares, Real Jardín Botánico, CSIC, Madrid, 2014.

[19] Lasso, C. A., Rial, A., Colonnello, G., MachadoAllison A., Trujillo, F., (Editores). XI. Humedales de la Orinoquia (Colombia- Venezuela), Serie Editorial Recursos Hidrobiológicos y Pesqueros Continentales de Colombia, Instituto de Investigación de Recursos Biológicos Alexander von Humboldt (IAvH), Bogotá, D.C., Colombia, 2014.

[20] Quiñonez Oquendo, L.E., \& Wagner Vega, K. Inventario de Plantas Acuáticas y de Ribera Asociadas al Río Toro Negro, Tributario del Río Grande de Manatí, San Juan, Puerto Rico: Departamento de Recursos Naturales y Ambientales, 2016.

[21] Schmidt-Mumm, U. Vegetación acuática y palustre de la parte alta de la hoya del río Namay (Albán, Cundinamarca), Perez-Arbelaezia, 2(6-7) 941, 1988.

[22] Posada, J.A., \& López, M.T. Plantas Acuáticas del Altiplano del Oriente Antioqueño, Medellín: Universidad Católica de Oriente, 2011.

[23]Aguirre, N. Hidrobiologia Sanitaria, Universidad de Antioquia, Medellin- Colombia, 2013.
[24] Fernández-Alaez, C., Fernández-Alaez, M., Santiago Ibarlucea, N.F., Núñez Labra, G., Aboal Sanjurjo, M. Id-Tax. Catálogo y claves de identificación de organismos del grupo macrófitos utilizados como elementos de calidad en las redes de control del estado ecológico, España, 2012.

[25] García, J.M., Sarmiento L.F., Salvador, M., Porras, L.S. Uso de bioindicadores para la evaluación de la calidad del agua en ríos: aplicación en ríos tropicales de alta montaña, Revisión Corta. UGCiencia, 23(1): 47-62, 2017.

[26] Ministerio de Agricultura, Alimentación y Medio Ambiente. Protocolo de muestreo y laboratorio de Macrófitos en ríos, España, 2015.

[27] Shannon, C.E., Weaver, W. The mathematical theory of communication, The University of Illinios Press, 1949.

[28] Simpson, E.H. Measurement of diversity, Nature, 163(4148): 688, 1949.

[29] Pielou, G.A. Ecological Diversity, Wiley New York, 1998.

[30] Legendre, P. \& H.J.B. Birks. From classical to canonical ordination. Chapter 8, 201-248, 2012

[31] Engloner, A.I, Szalma, E., Sipos, K., Dinka, M. Occurrence and habitat preference of aquatic macrophytes in a large river channel, Community Ecology 14(2): 243-248, 2013.

[32] Hrivnák, R., Ot’ahel'ová, H., Gömöry, D. Seasonal dynamics of macrophyte abundance in two regulated streams, Cent. Eur. J. Biol, 4(2):241249, 2009.

[33] Abati, S., Minciardi, M.R., Ciadamidaro, S., Fattorini, S., Ceschin, S. Response of macrophyte communities to flow regulation in mountain streams, Environ. Monit. Assess, 2016.

[34] Vukov, D., Ilić, M., Ćuk, M., Radulović, S., Igić, R., Janauer, G.A. Combined effects of physical environmental conditions and anthropogenic alterations are associated with macrophyte habitat fragmentation in rivers - Study of the Danube in Serbia, Science of the Total Environment, 634:780790, 2018.

[35] Manolaki, P., Papastergiadou, E. Environmental factors influencing macrophytes 
assemblages in a middle-sized Mediterranean stream, River res. Applic, 2015

[36] Arocena, R., Mazzeo, N. Macrófitas acuáticas de un arroyo urbano en Uruguay: Su relación con la calidad del agua, Rev. Bio. Trop, 42(3):723-728, 1994.

[37] Manolaki, P., Papastergiadou, E. The impact of environmental factors on the distribution pattern of aquatic macrophytes in a middle-sized Mediterranean stream, Aquatic Botany, 104: 34-46, 2013.

[38] Álvarez, M., Ramírez, C., Deil, U. Ecología y distribución de Hydrocotyle cryptocarpa speg. En Sudamérica, Gayana Bot, 65(2):139-144, 2008.

[39] Alfonso, G.L., Prina, A.O., Muiño, W.A. Una nueva especie del género Oxalis (Oxalidaceae) para la provincia de Mendoza, Argentina, (San Isidro), Hickenia, 3(51):213-216, 2004.

[40] Tomás, P., Moreno, J.L., Aboal, M., Oscoz, J., Durán, C., Navarro, P. Evaluación del estado ecológico de los ríos de la cuenca del río Ebro mediante el índice trófico de macrófitos IVAM-G (Índice de Vegetación Acuática Macroscópica), Limnetica, 35(1): 219-234, 2016.

[41] Lagos-López, M.I., Sáenz-Jiménez, F.A., Morales-Puentes, M.E. Briófitos reófilos de tres quebradas del páramo de Mamapacha, Chinavita (Boyacá-Colombia), Acta Biológica Colombiana, 13(1):143-160, 2008.

[42] García Martinez, S., Basilio B, H., Herazo V, F., Mercado G, J. \& Morales P, M. Diversidad de briófitos en los Montes de María, Colosó (Sucre, Colombia), Colombia Forestal, 19(1), 41-52, 2016.

[43] Chambers, P.A., Lacoul, P., Murphy, K.J., Thomaz, S.M. Global diversity of aquatic macrophytes in freshwater, Rev. Hydrobiologia, 595:9-26, 2008.

[44] Villareal Q., J.A., Carranza P., M.A., Estrada C., E., Rodríguez G., A. Flora riparia de los ríos Sabinas y San Rodrigo, Coahuila, México, Acta Biológica Mexicana. 75:1-20, 2006. 